\title{
Sphingosine kinase 1 enhances the invasion and migration of non-small cell lung cancer cells via the AKT pathway
}

\author{
LIANGMING ZHU ${ }^{1}$, ZHOU WANG $^{2}$, YUXIA LIN ${ }^{1}$, ZHITAO CHEN $^{1}$, HAIBO LIU $^{1}$, \\ YING CHEN $^{1}$, NINGNING WANG ${ }^{3}$ and XIUE SONG ${ }^{3}$ \\ ${ }^{1}$ Department of Thoracic Surgery, Jinan Central Hospital, Shandong University, Jinan, Shandong 250013; \\ ${ }^{2}$ Department of Thoracic Surgery, Shandong Province Hospital, Shandong University, Jinan, Shandong 250013; \\ ${ }^{3}$ Department of Gynaecology and Obstetrics, Jinan Central Hospital, Shandong University, Jinan, Shandong 250013, P.R. China
}

Received July 20, 2014; Accepted October 3, 2014

DOI: $10.3892 /$ or.2014.3683

\begin{abstract}
Sphingosine kinase 1 (SphK1) has been shown to play an important role in the progression of a number of human cancers. It has been reported that the expression of SphK1 is greatly elevated in non-small cell lung cancer (NSCLC) tissues. However, its role and underlying mechanisms in NSCLC have not been fully elucidated. In the present study, we found that SphK1 was highly expressed in NSCLC cells. Overexpression of SphK1 promoted the invasion and migration of NSCLC cells, while knockdown of SphK1 suppressed the invasion and migration. Furthermore, we demonstrated that SphK1 decreased the protein level of E-cadherin, yet increased the protein level of Snail. In addition, SphK1 was able to stimulate the activation of AKT. Inhibition of the AKT pathway attenuated the biological functions of NSCLC cells induced by overexpression of SphK1. Taken together, our findings suggest that SphK1 can enhance the invasion and migration of NSCLC cells via activation of the AKT pathway and regulation of E-cadherin and Snail expression. Thus, SphK1 could be a potential target for the detection and treatment of NSCLC.
\end{abstract}

\section{Introduction}

Lung cancer is one of the most common malignancies worldwide, and is the leading cause of cancer-related mortality among both men and women (1). Lung cancer can be divided into two main subtypes: small cell lung cancer (SCLC) and non-small cell lung cancer (NSCLC). NSCLC accounts for $\sim 85 \%$ of lung cancer cases. Most patients with NSCLC are typically at an advanced stage when diagnosed (2). Therefore, uncovering the molecular mechanisms of NSCLC and identifying new biomarkers may be crucial for the diagnosis and treatment of NSCLC.

Correspondence to: Dr Xiue Song, Department of Gynaecology and Obstetrics, Jinan Central Hospital, Shandong University, $105 \mathrm{Jie}$ Fang Road, Jinan, Shandong 250013, P.R. China

E-mail: xiuesong@163.com

Key words: SphK1, invasion, migration, non-small cell lung cancer, EMT, AKT
Sphingosine kinase (SphK) is a type of conserved lipid kinase that can convert phosphorylation of sphingosine to sphingosine-1-phosphate (S1P). S1P is an important bioactive lipid mediator that regulates various aspects of cellular processes during tumorigenesis $(3,4)$. Thus, SphK may also play a crucial role in the progression of cancer. There are two functional SphK isoforms [sphingosine kinase 1 (SphK1; and SphK2)] that have been identified and characterized in mammalian cells (5). As one member of the SphK family, SphK1 activity can be stimulated by a series of cytokines such as EGF and VEGF (6), and acts as an oncogenic enzyme in tumor cells (7). Overexpression of SphK1 has been demonstrated in many cancer types including breast, prostate, gastric and colon cancer, and is closely related with cancer progression and the poor survival of patients (8-11). Many reports have proved that SphK1 participates in the regulation of tumor cell antiapoptosis, growth and survival $(12,13)$. Studies also show that SphK1 promotes breast cancer progression by stimulating angiogenesis and lymphangiogenesis (14), and is involved in the invasion and metastasis of esophageal carcinoma (15). The expression of SphK1 is significantly increased in NSCLC, and overexpression of SphK1 promotes tumor progression by enhancing resistance to apoptosis (16). However, whether SphK1 participates in the invasion and metastasis processes of NSCLC has not been previously reported.

In the present study, we examined the expression of SphK1 in different NSCLC cell lines. We also investigated the role of SphK1 in NSCLC cell invasion and migration, and attempted to reveal the underlying mechanisms.

\section{Materials and methods}

Reagents and antibodies. LY294002 was obtained from Sigma (St. Louis, MO, USA). Antibodies against SphK1, $\beta$-actin and E-cadherin were purchased from Santa Cruz Biotechnology (Santa Cruz, CA, USA). Antibodies against Snail, phospho-AKT and AKT were purchased from Cell Signaling Technology (Danvers, MA, USA).

Cell lines and culture conditions. All cell lines used in our experiments were purchased from the cell bank of the Chinese Academy of Sciences (Shanghai, China). The non-malignant 
lung epithelial cell line BEAS-2B and the NSCLC cell lines H460, HCC827, H1299 and A549 were all cultured in DMEM (Gibco, Grand Island, NY, USA) supplement with $10 \%$ fetal bovine serum (FBS) in a humidified atmosphere containing $5 \% \mathrm{CO}_{2}$ at $37^{\circ} \mathrm{C}$.

Real-time PCR. Total RNA was extracted using TRIzol reagent (Invitrogen, Carlsbad, CA, USA), according to the manufacturer's instructions. Total RNA was measured by NanoDrop 2000 (Thermo Scientific, Waltham, MA, USA) and then $2 \mu \mathrm{g}$ of total RNA was reverse transcribed into cDNA using a cDNA synthesis kit (Promega Corporation, Madison, WI, USA). Real-time PCR was performed with the primers of SphK1 (sense, 5'-GGCTGCTGTCACCCATGAA-3' and antisense, 5'-TCACTCTCTAGGTCCACATCAG-3'); or $\beta$-actin (sense, 5'-TGAGCGCGGCTACAGCTT-3' and antisense, 5'-TCCTTAATGTCACGCACGATTT-3'), respectively. The real-time PCR thermal cycle conditions were as follows: $10 \mathrm{~min}$ at $95^{\circ} \mathrm{C}, 40$ cycles of $15 \mathrm{sec}$ at $95^{\circ} \mathrm{C}$ and $1 \mathrm{~min}$ at $60^{\circ} \mathrm{C}$. Finally, the relative gene expression level of SphK1 was normalized to $\beta$-actin expression, and then calculated using the $2^{-\Delta \Delta \mathrm{Ct}}$ method.

Western blotting. Cell lysate was extracted using RIPA buffer containing protease inhibitors and phosphatase inhibitors (Applygen Technologies Inc., Beijing, China). The concentration of total protein was evaluated by the BCA method. Equal amounts of protein were separated using SDS-PAGE gel and then electroblotted onto a PVDF membrane. The PVDF membrane was blocked with 5\% BSA diluted in TBST [10 $\mathrm{mM}$ Tris (pH 7.4), $100 \mathrm{mM} \mathrm{NaCl}$ and 5\% Tween-20], and then the membrane was incubated separately with primary antibodies against SphK1 (1:500), E-cadherin (1:500), Snail $(1: 1,000)$, phospho-AKT $(1: 1,000)$, AKT $(1: 1,000)$ or $\beta$-actin $(1: 1,000)$ at $4^{\circ} \mathrm{C}$ overnight. Next, the membrane was incubated with peroxidase-conjugated secondary antibody (Santa Cruz Biotechnology) for $1 \mathrm{~h}$ at room temperature. The bands were visualized using an enhanced chemiluminescence (ECL) kit (Applygen Technologies Inc.). After exposure to X-ray film, the densitometry was quantified using Quantity One software (Bio-Rad, Hercules, CA, USA).

Cell transfections. For overexpression of SphK1, a pcDNA3.1 vector encoding full-length SphK1 was constructed and obtained from GenePharma Co., Ltd. (Shanghai, Beijing). An empty pcDNA3.1 vector was used as the negative control (NC). A549 cells were transfected with the SphK1 vector or NC vector, respectively, using Lipofectamine ${ }^{\mathrm{TM}} 2000$ reagent (Life Technologies Co., Carlsbad, CA, USA) according to the manufacturer's instructions. Cells were used $48 \mathrm{~h}$ later for the following experiments.

For knockdown of SphK1, a siRNA targeting SphK1 was used with the sequence as follows: SphK1 siRNA, 5'-GCAGGCAUAUGGAGUAUGA-3'. A scramble siRNA was used as the negative control. A549 cells were transfected with the SphK1 siRNA (siSphK1) or the control siRNA (siCtrl), respectively, using Lipofectamine ${ }^{\mathrm{TM}} 2000$ reagent. Forty-eight hours later, the cells were used in the following experiments.

Invasion assay. Cell invasion ability was analyzed using a 24-well Transwell chamber, which contained $8-\mu \mathrm{m}$ pore size
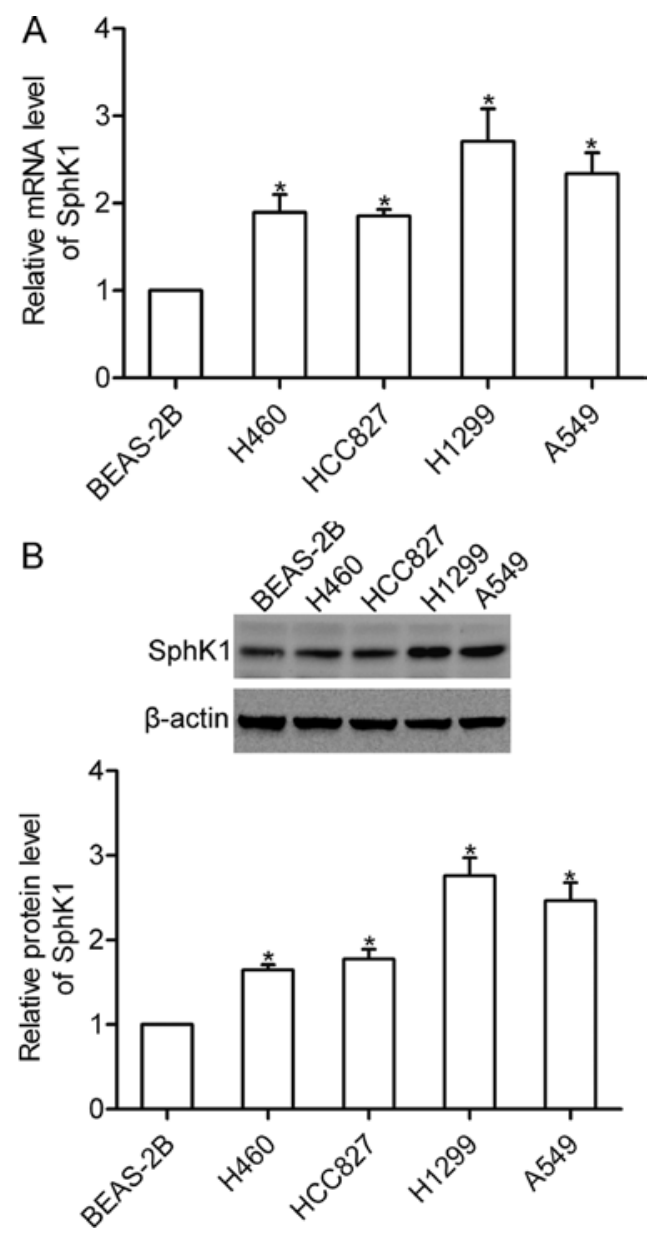

Figure 1. Expression of SphK1 in NSCLC cells. (A) The mRNA expression of SphK1 in BEAS-2B, H460, HCC827, H1299 and A549 cells was observed by real-time PCR. (B) The protein level of SphK1 in BEAS-2B, H460, HCC827, H1299 and A549 cells was detected by western blot analysis. "p<0.05. SphK1, sphingosine kinase 1; NSCLC, non-small cell lung cancer.

polyethylene membranes (Costar, San Diego, CA, USA). In brief, $1.0 \times 10^{5}$ cells in $0.2 \mathrm{ml}$ medium supplement with $1 \%$ FBS were placed into the upper chamber, which was coated with $50 \mu 11 \mu \mathrm{g} / \mathrm{ml}$ Matrigel before being used. The lower chamber was filled with $600 \mu \mathrm{l}$ medium supplement with $30 \%$ FBS. Then the chambers were incubated for $16 \mathrm{~h}$ at $37^{\circ} \mathrm{C}$ in a humidified atmosphere containing $5 \% \mathrm{CO}_{2}$. Cells that penetrated through the Matrigel-coated membrane were stained with crystal violet after fixation in $4 \%$ formaldehyde, and then photographed at $\times 200$ magnification under a light microscope. Cell number was counted from seven random fields, and the mean number was calculated to indicate cell invasive ability.

Migration assay. Cell migration ability was also evaluated in a 24-well Transwell chamber model. Briefly, the upper chamber was seeded with $1 \times 10^{5}$ cells in $0.2 \mathrm{ml}$ medium supplement with $1 \%$ FBS and the lower chamber was filled with $600 \mu 1$ medium supplement with $30 \%$ FBS as a chemoattractant. After incubation for $16 \mathrm{~h}$ at $37^{\circ} \mathrm{C}$, cells on the upper chamber were removed with a cotton swab, and cells that migrated through the membrane were stained with crystal violet. Finally, the migrated cells were observed under a light microscope at x200 magnification, and the average cell number was determined from seven random fields. 

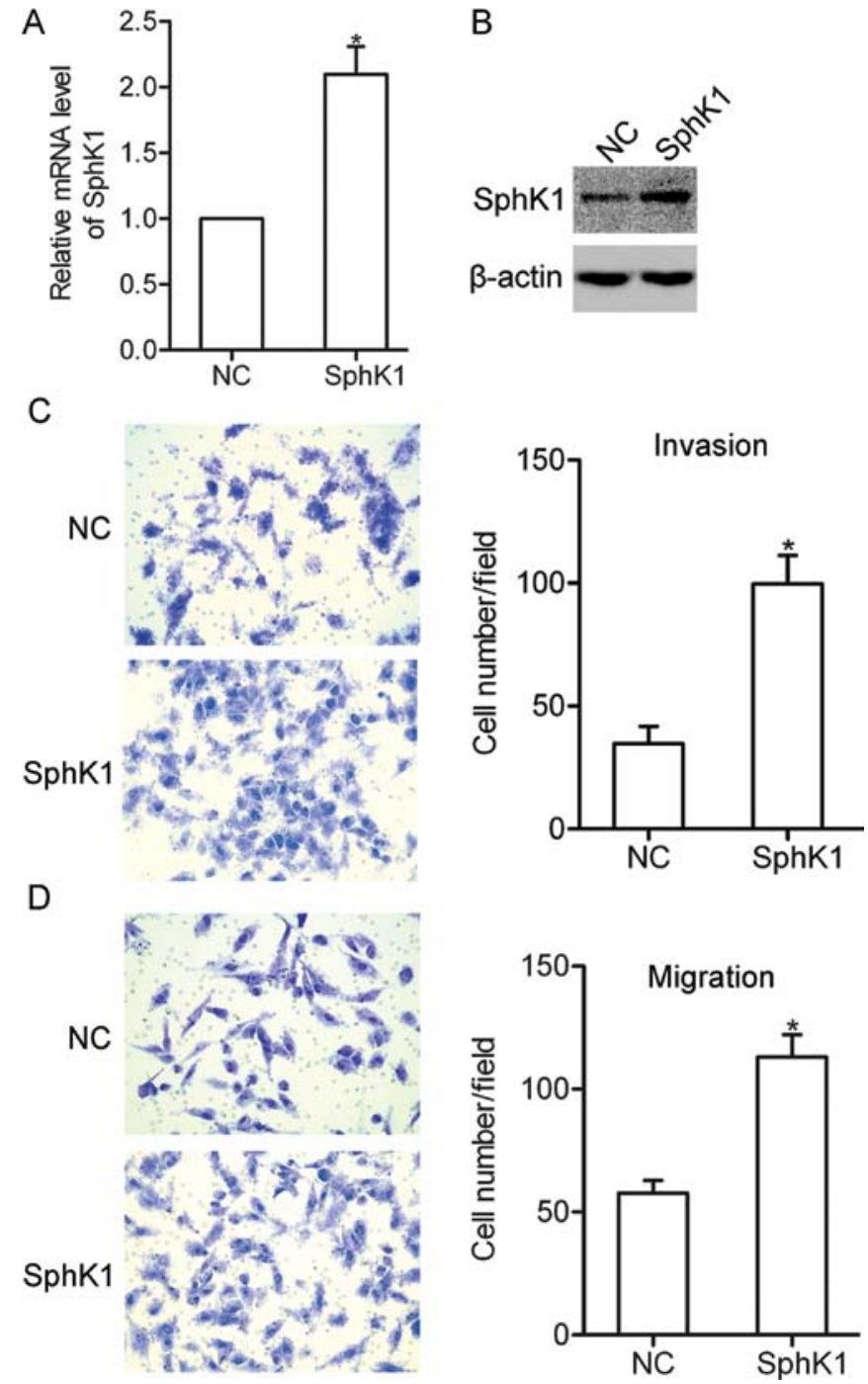

Figure 2. Overexpression of SphK1 promotes the invasion and migration of A549 cells. A549 cells were transfected with the SphK1 vector (denoted as SphK1) or an empty vector (denoted as NC), respectively. (A) The mRNA level of SphK1 was detected by real-time PCR. (B) The protein level of SphK1 was detected by western blot analysis. (C) Effect of SphK1 overexpression on the invasion of A549 cells. (D) Effect of SphK1 overexpression on the migration of A549 cells. ${ }^{*} \mathrm{p}<0.05$. SphK1, sphingosine kinase 1 ; NC, negative control.

Statistical analysis. All experiments were performed at least three times. The data were analyzed using the software package of SPSS 17.0, and data are presented as means \pm standard deviation. The significance of the difference between two groups was assessed by the Student's t-test. Differences were considered to indicate a statistically significant result at $\mathrm{p}<0.05$.

\section{Results}

SphK1 is highly expressed in NSCLC cells. Using real-time PCR, we firstly examined the mRNA expression of SphK1 in the NSCLC cell lines H460, HCC827, H1299 and A549 as well as in the non-malignant lung epithelial cell line BEAS2B. The results showed that the mRNA expression of SphK1 was significantly expressed in the NSCLC cells (Fig. 1A). Furthermore, the protein level of SphK1 was detected using
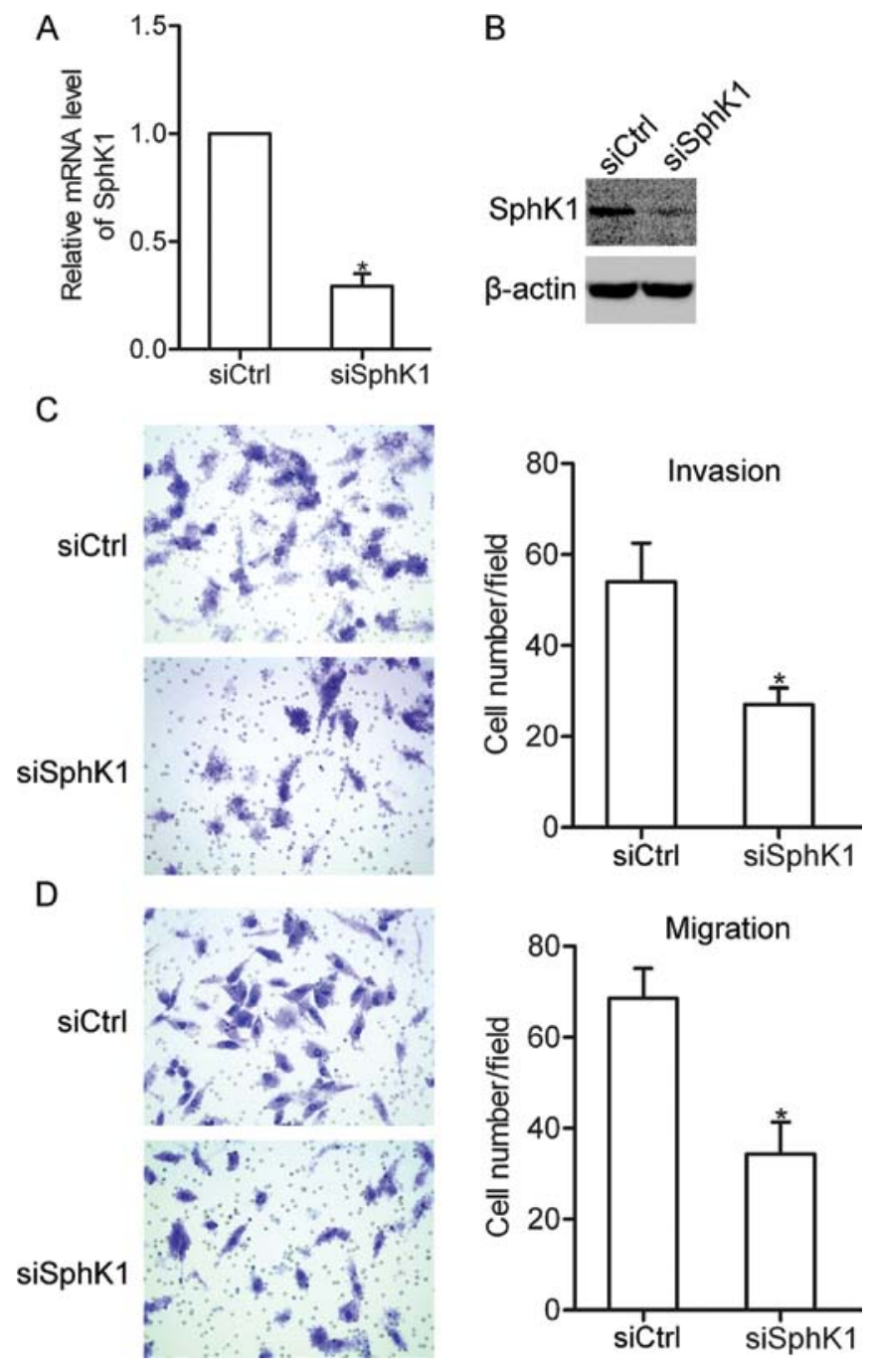

Figure 3. Knockdown of SphK1 inhibits the invasion and migration of A549 cells. A549 cells were transfected with SphK1 siRNA (denoted as siSphK1) or a scramble control siRNA (denoted as siCtrl), respectively. (A) The mRNA level of SphK1 was detected by real-time PCR. (B) The protein level of SphK1 was detected by western blot analysis. (C) Effect of SphK1 knockdown on the invasion of A549 cells. (D) Effect of SphK1 knockdown on the migration of A549 cells. * p<0.05. SphK1, sphingosine kinase 1; siSphK1, SphK1 siRNA; siCtrl, control siRNA.

western blotting, and the results showed that SphK1 protein was highly expressed in the NSCLC cells (Fig. 1B).

Overexpression of SphK1 promotes NSCLC cell invasion and migration. To investigate the role of SphK1 in the biological behavior of NSCLC cells, SphK1 was overexpressed in the A549 cells by transfection with a pcDNA 3.1-SphK1 vector (Fig. 2A and B). Using invasion and migration assays, we found that overexpression of SphK1 promoted the invasion and migration of the A549 cells (Fig. 2C and D), suggesting that $\mathrm{SphK} 1$ is involved in the invasion and migration of NSCLC cells.

Knockdown of SphK1 inhibits NSCLC cell invasion and migration. To further characterize the effect of SphK1 on NSCLC cells, SphK1 siRNA was used to suppress the expression of SphK1 in the A549 cells (Fig. 3A and B). The results showed 
A
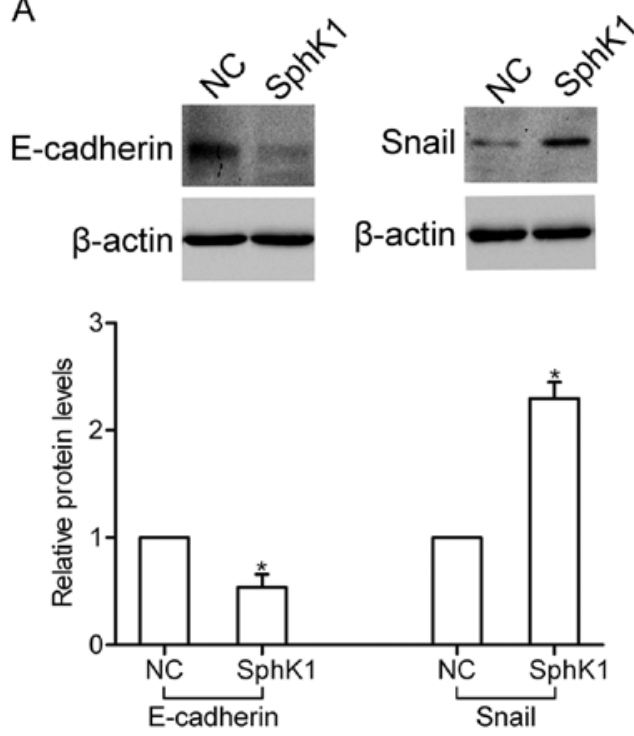

B
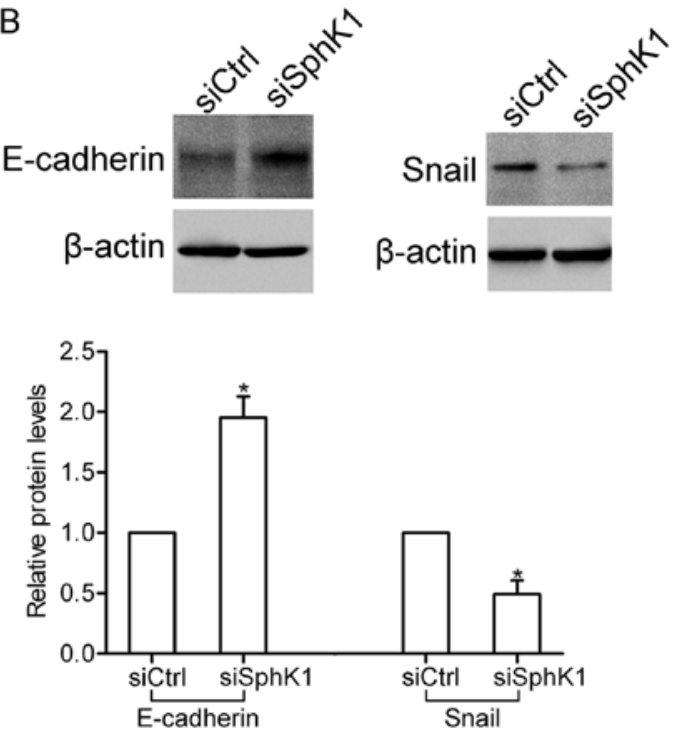

Figure 4. Involvement of SphK1 in the regulation of E-cadherin and Snail expression. (A) Overexpression of SphK1 decreased the E-cadherin level, but increased the Snail level. (B) Knockdown of SphK1 increased the E-cadherin level, but decreased the Snail level. *p<0.05. SphK1, sphingosine kinase 1.
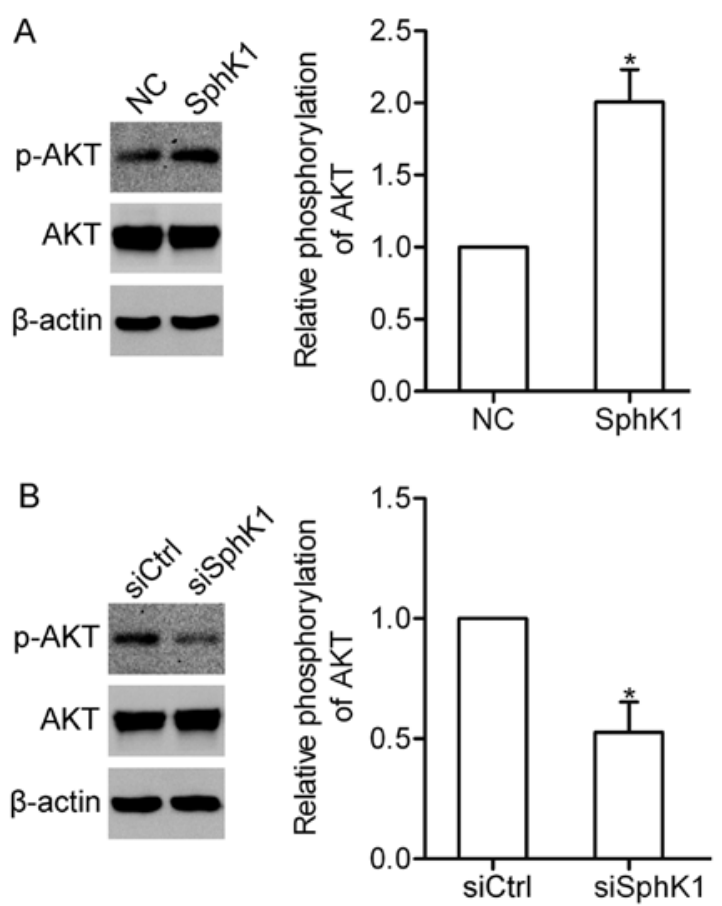

Figure 5. SphK1 stimulates the activation of AKT in A549 cells. (A) Overexpression of SphK1-induced the activation of AKT in the A549 cells (B) Knockdown of SphK1 attenuated the activation of AKT in the A549 cells. "p<0.05. SphK1, sphingosine kinase 1.

that transfection of SphK1 siRNA greatly suppressed the invasion and migration of the A549 cells (Fig. 3C and D). These data further confirm the involvement of SphK1 in NSCLC cell invasion and migration.

SphK1 participates in the regulation of several EMT-related genes. The epithelial-mesenchymal transition (EMT) plays a crucial role in the invasion and metastasis of NSCLC cells. Here, western blot analysis showed that overexpression of
SphK1 decreased the expression of E-cadherin, yet increased the expression of Snail (Fig. 4A). In contrast, knockdown of SphK1 enhanced the expression of E-cadherin, yet inhibited the expression of Snail (Fig. 4B). These results suggest that SphK1 participates in the regulation of E-cadherin and Snail expression.

SphK1 contributes to the activation of AKT in NSCLC cells. It has been demonstrated that SphK1 can activate the PI3K/AKT pathway in glioma cells (17). We here wondered whether SphK1 could affect the AKT pathway in NSCLC cells. Overexpression of SphK1 induced the activation of AKT in the A549 cells (Fig. 5A). In contrast, knockdown of SphK1 attenuated the activation of AKT (Fig. 5B). The data indicate that SphK1 participates in the activation of the AKT pathway.

The AKT pathway is involved in SphK1-enhanced invasion and migration. To determine whether the AKT pathway is associated with the SphK1-enhanced invasion and migration, LY294002 $(10 \mu \mathrm{M})$ was used to block the AKT pathway in the A549 cells. Using invasion and migration assays, we found that overexpression of SphK1 promoted the invasion and migration. However, the SphK1-enhanced invasion and migration was greatly suppressed due to inhibition of the AKT pathway (Fig. 6A and B). These results suggest that the AKT pathway is required for SphK1-enhanced invasion and migration.

SphK1 regulates the expression of EMT-related genes via the AKT pathway. We further examined the role of the AKT pathway in the SphK1-mediated E-cadherin and Snail expression. Notably, the results showed that overexpression of SphK1 decreased the expression of E-cadherin, yet increased the expression of Snail in the dimethyl sulfoxide (DMSO)-treated group. Nevertheless, after inhibition of the AKT pathway by LY294002 (10 $\mu \mathrm{M})$, the SphK1-mediated expression changes 

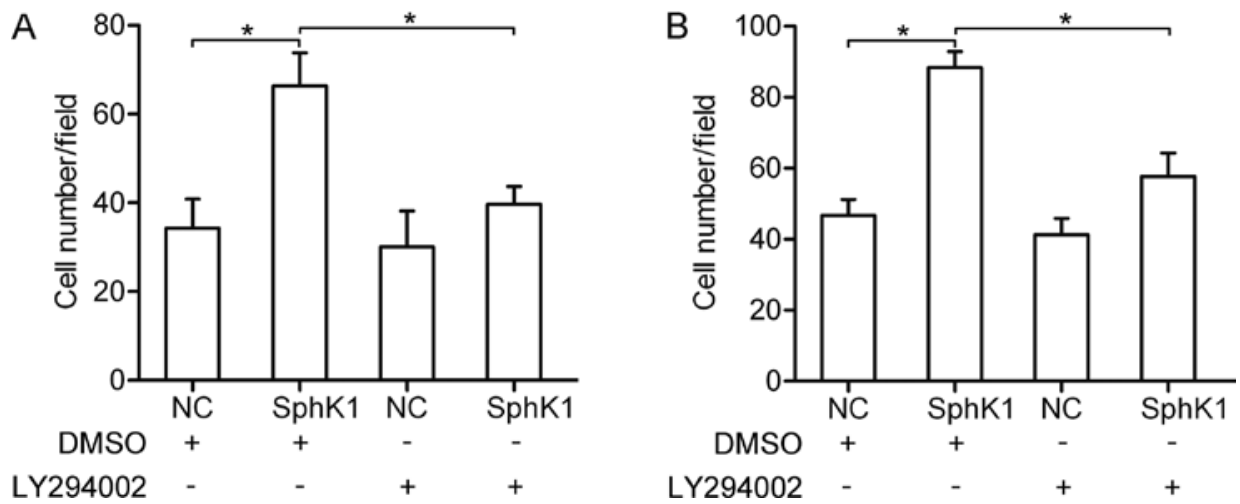

Figure 6. Effects of AKT pathway on the SphK1-mediated invasion and migration. A549 cells were pretreated with LY294002 (10 $\mu$ M) or DMSO for 30 min. Then, (A) invasion assay and (B) migration assay were performed in A549 cells. " $\mathrm{p}<0.05$. SphK1, sphingosine kinase 1; DMSO, dimethyl sulfoxide.
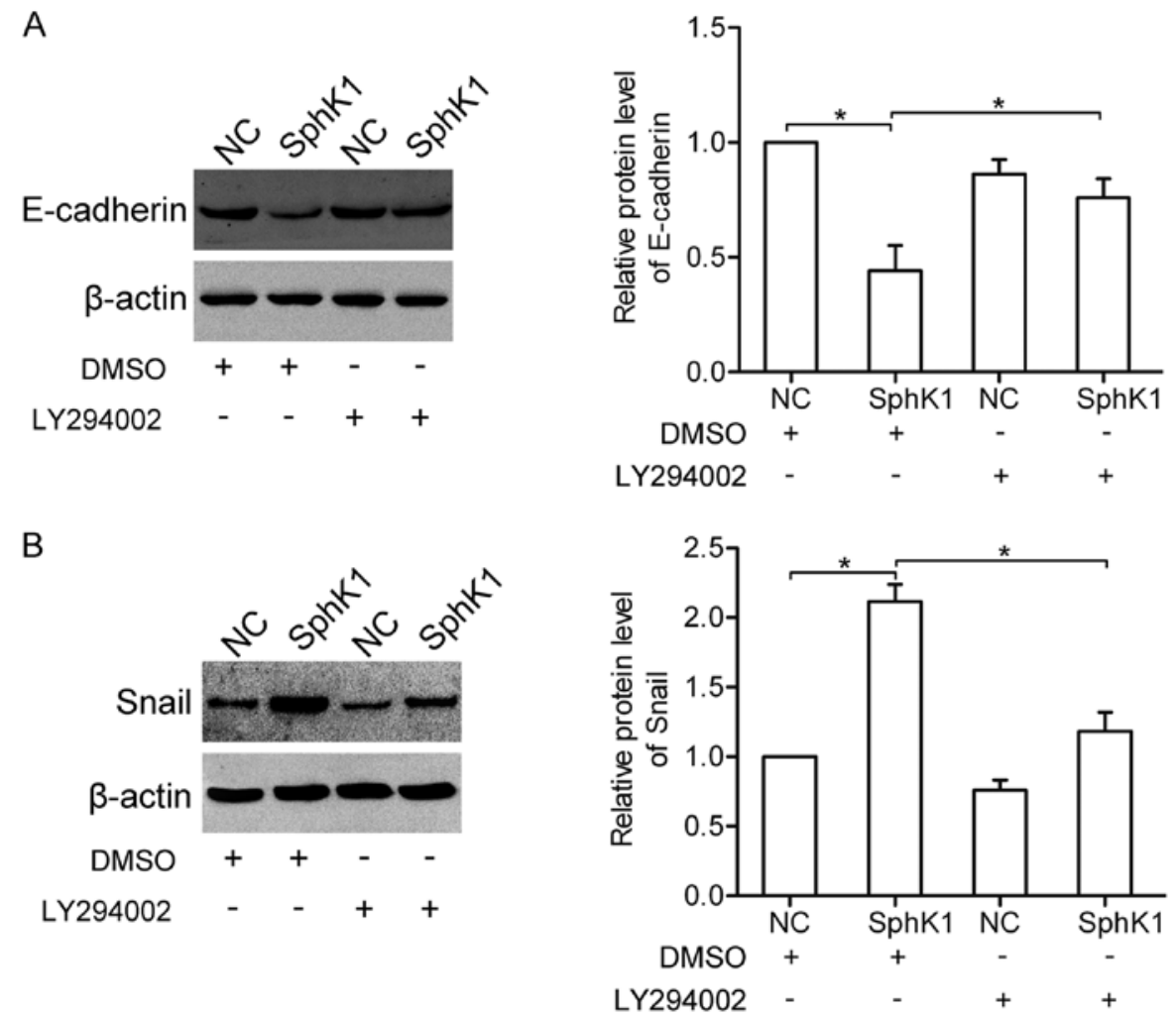

Figure 7. Effects of the AKT pathway on the SphK1-mediated expression changes in E-cadherin and Snail. A549 cells were treated with LY294002 (10 $\mu$ M) or DMSO for $16 \mathrm{~h}$. Western blotting was performed to detect the protein levels of (A) E-cadherin and (B) Snail. "p $<0.05$. SphK1, sphingosine kinase 1; DMSO, dimethyl sulfoxide.

in E-cadherin and Snail were attenuated (Fig. 7A and B), suggesting that SphK1 regulates the expressions of E-cadherin and Snail via the AKT pathway.

\section{Discussion}

As one important member of the SphK family, SphK1 plays a crucial role in the regulation of intercellular and intracellular signaling (18). Overexpression of SphK1 has been observed in diverse tumors (19-21). It is reported that the expression of SphK1 is markedly increased in NSCLC tissues, and is correlated with tumor progression and poor survival of patients with NSCLC (16). In the present study, we found that the expres- sion of SphK1 was elevated in all detected NSCLC cells as compared to normal lung epithelial BEAS-2B cells, indicating a role of SphK1 in the progression of NSCLC cells.

SphK1 has been reported to act as a regulator in diverse cellular processes in tumor. It is well known that SphK1 plays a positive role in regulating the proliferation of tumor cells, including breast, prostate and thyroid cancer cells $(13,22,23)$. Studies have also demonstrated that SphK1 contributes to the apoptosis resistance in glioma and NSCLC cells $(16,17)$, and promotes tumor progression of colon cancer (24). In addition, SphK1 is required for EGF-directed motility in breast cancer MCF-7 cells, and promotes breast cancer progression by stimulating angiogenesis and lymphangiogenesis $(14,25)$. 
Further studies show that SphK1 accounts for the invasion and migration of colon cancer, hepatocellular carcinoma and ovarian cancer cells (26-28), suggesting the possible involvement of SphK1 in tumor metastasis. In the present study, we investigated the role of SphK1 in NSCLC cells. We found that overexpression of SphK1 greatly enhanced the invasion and migration of NSCLC cells. In contrast, knockdown of SphK1 significantly inhibited the invasion and migration. All of our findings further confirm the notion that SphK1 is one of the key modulators in NSCLC invasion and metastasis.

Accumulating evidence indicates that SphK1 participates in the activation of multiple signaling pathways in tumor cells. It is reported that SphK1 contributes to the regulation of CD44 protein expression through the ERK signaling pathway (29), and enhances colon cancer cell proliferation and invasiveness via activation of ERK1/2 and suppression of p38 MAPK pathways (26). In addition, SphK1 enhances resistance to apoptosis through activation of the Akt/FOXO3a/Bim pathway in glioma cells (17), and blockage of SphK1 inhibits Akt signaling in human glioblastoma cells and xenografts (30). In the present study, our results showed that overexpression of SphK1 stimulated the activation of AKT, whereas knockdown of SphK1 attenuated the activation of AKT in A549 cells, suggesting that SphK1 contributes to the activation of the AKT pathway in NSCLC cells. The AKT pathway is frequently dysregulated in tumors, and plays a pivotal role in tumor invasion and metastasis (31). Here, we found that inhibition of the AKT pathway attenuated the invasion and migration induced by overexpression of SphK1, suggesting that the AKT pathway is required for SphK1-mediated invasion and migration.

The process of EMT is considered to be necessary for the acquisition of increased motility and invasiveness of cancer cells. E-cadherin, which is regulated by transcription factors such as Snail and Slug, plays a crucial role in the maintenance of cell polarity by mediating cell-cell adherence (32). However, whether SphK1 can affect the expression of these EMT-related genes is still unclear. In the present study, we found that SphK1 decreased the protein level of E-cadherin, but increased the protein level of Snail. Furthermore, blockage of the AKT pathway attenuated the SphK1-mediated expression changes in above EMT-related genes. Thus, it is possible that SphK1 can regulate the EMT process via the AKT pathway in NSCLC cells.

In conclusion, our findings demonstrated that SphK1 increases the E-cadherin protein level but decreases Snail protein level via the AKT pathway, which then participates in the invasion and migration of NSCLC cells. Therefore, targeting SphK1 may provide new strategies for the therapeutics of NSCLC.

\section{References}

1. Siegel R, Ma J, Zou Z and Jemal A: Cancer statistics, 2014. CA Cancer J Clin 64: 9-29, 2014.

2. William WN Jr, Lin HY, Lee JJ, Lippman SM, Roth JA and Kim ES: Revisiting stage IIIB and IV non-small cell lung cancer: analysis of the Surveillance, Epidemiology, and End Results data. Chest 136: 701-709, 2009.

3. Taha TA, Hannun YA and Obeid LM: Sphingosine kinase: biochemical and cellular regulation and role in disease. J Biochem Mol Biol 39: 113-131, 2006.

4. Spiegel S and Milstien S: Sphingosine-1-phosphate: an enigmatic signalling lipid. Nat Rev Mol Cell Biol 4: 397-407, 2003.
5. Liu H, Chakravarty D, Maceyka M, Milstien S and Spiegel S: Sphingosine kinases: a novel family of lipid kinases. Prog Nucleic Acid Res Mol Biol 71: 493-511, 2002.

6. Shida D, Takabe K, Kapitonov D, Milstien S and Spiegel S: Targeting SphK1 as a new strategy against cancer. Curr Drug Targets 9: 662-673, 2008.

7. Cuvillier O, Ader I, Bouquerel P, et al: Activation of sphingosine kinase-1 in cancer: implications for therapeutic targeting. Curr Mol Pharmacol 3: 53-65, 2010.

8. Li W, Yu CP, Xia JT, et al: Sphingosine kinase 1 is associated with gastric cancer progression and poor survival of patients. Clin Cancer Res 15: 1393-1399, 2009.

9. Tan SS, Khin LW, Wong L, et al: Sphingosine kinase 1 promotes malignant progression in colon cancer and independently predicts survival of patients with colon cancer by competing risk approach in South Asian population. Clin Transl Gastroenterol 5: e51, 2014.

10. Malavaud B, Pchejetski D, Mazerolles C, et al: Sphingosine kinase-1 activity and expression in human prostate cancer resection specimens. Eur J Cancer 46: 3417-3424, 2010.

11. Ruckhäberle E, Rody A, Engels K, et al: Microarray analysis of altered sphingolipid metabolism reveals prognostic significance of sphingosine kinase 1 in breast cancer. Breast Cancer Res Treat 112: 41-52, 2008.

12. Yang YL, Ji C, Cheng L, et al: Sphingosine kinase-1 inhibition sensitizes curcumin-induced growth inhibition and apoptosis in ovarian cancer cells. Cancer Sci 103: 1538-1545, 2012.

13. Dayon A, Brizuela L, Martin C, et al: Sphingosine kinase-1 is central to androgen-regulated prostate cancer growth and survival. PLoS One 4: e8048, 2009.

14. Nagahashi M, Ramachandran S, Kim EY, et al: Sphingosine-1phosphate produced by sphingosine kinase 1 promotes breast cancer progression by stimulating angiogenesis and lymphangiogenesis. Cancer Res 72: 726-735, 2012.

15. Pan J, Tao YF, Zhou Z, et al: A novel role of sphingosine kinase-1 (SPHK1) in the invasion and metastasis of esophageal carcinoma. J Transl Med 9: 157, 2011.

16. Song L, Xiong H, Li J, et al: Sphingosine kinase-1 enhances resistance to apoptosis through activation of PI3K/Akt/NF- $\mathrm{KB}$ pathway in human non-small cell lung cancer. Clin Cancer Res 17: 1839-1849, 2011.

17. Guan H, Song L, Cai J, et al: Sphingosine kinase 1 regulates the Akt/FOXO3a/Bim pathway and contributes to apoptosis resistance in glioma cells. PLoS One 6: e19946, 2011.

18. Pyne S, Lee SC, Long J and Pyne NJ: Role of sphingosine kinases and lipid phosphate phosphatases in regulating spatial sphingosine 1-phosphate signalling in health and disease. Cell Signal 21: 14-21, 2009.

19. Meng XD, Zhou ZS, Qiu JH, Shen WH, Wu Q and Xiao J: Increased SPHK1 expression is associated with poor prognosis in bladder cancer. Tumour Biol 35: 2075-2080, 2014.

20. Bayerl MG, Bruggeman RD, Conroy EJ, et al: Sphingosine kinase 1 protein and mRNA are overexpressed in non-Hodgkin lymphomas and are attractive targets for novel pharmacological interventions. Leuk Lymphoma 49: 948-954, 2008.

21. Fuereder T, Hoeflmayer D, Jaeger-Lansky A, et al: Sphingosine kinase 1 is a relevant molecular target in gastric cancer. Anticancer Drugs 22: 245-252, 2011.

22. Nava VE, Hobson JP, Murthy S, Milstien S and Spiegel S: Sphingosine kinase type 1 promotes estrogen-dependent tumorigenesis of breast cancer MCF-7 cells. Exp Cell Res 281: 115-127, 2002.

23. Guan H, Liu L, Cai J, et al: Sphingosine kinase 1 is overexpressed and promotes proliferation in human thyroid cancer. Mol Endocrinol 25: 1858-1866, 2011.

24. Liu SQ, Su YJ, Qin MB, Mao YB, Huang JA and Tang GD: Sphingosine kinase 1 promotes tumor progression and confers malignancy phenotypes of colon cancer by regulating the focal adhesion kinase pathway and adhesion molecules. Int J Oncol 42: 617-626, 2013.

25. Sarkar S, Maceyka M, Hait NC, et al: Sphingosine kinase 1 is required for migration, proliferation and survival of MCF-7 human breast cancer cells. FEBS Lett 579: 5313-5317, 2005.

26. Liu SQ, Huang JA, Qin MB, et al: Sphingosine kinase 1 enhances colon cancer cell proliferation and invasion by upregulating the production of MMP-2/9 and uPA via MAPK pathways. Int $\mathbf{J}$ Colorectal Dis 27: 1569-1578, 2012.

27. Bao M, Chen Z, Xu Y, et al: Sphingosine kinase 1 promotes tumour cell migration and invasion via the S1P/EDG1 axis in hepatocellular carcinoma. Liver Int 32: 331-338, 2012. 
28. Zhang $\mathrm{H}$, Wang Q, Zhao Q and Di W: MiR-124 inhibits the migration and invasion of ovarian cancer cells by targeting SphK1. J Ovarian Res 6: 84, 2013.

29. Kawahara S, Otsuji Y, Nakamura M, et al: Sphingosine kinase 1 plays a role in the upregulation of CD44 expression through extracellular signal-regulated kinase signaling in human colon cancer cells. Anticancer Drugs 24: 473-483, 2013.

30. Kapitonov D, Allegood JC, Mitchell C, et al: Targeting sphingosine kinase 1 inhibits Akt signaling, induces apoptosis, and suppresses growth of human glioblastoma cells and xenografts. Cancer Res 69: 6915-6923, 2009.
31. Jiang H, Gao M, Shen Z, et al: Blocking PI3K/Akt signaling attenuates metastasis of nasopharyngeal carcinoma cells through induction of mesenchymal-epithelial reverting transition. Oncol Rep 32: 559-566, 2014.

32. Yilmaz M and Christofori G: EMT, the cytoskeleton, and cancer cell invasion. Cancer Metastasis Rev 28: 15-33, 2009. 